\title{
Gouvernances quotidiennes au cœur des structures de santé. Les cas d'Orodara et de Banfora, Burkina
} Faso

\section{Fatoumata Ouattara}

\section{CpenEdition}

\section{Journals}

Édition électronique

URL : http://journals.openedition.org/apad/145

DOI : 10.4000/apad. 145

ISSN : 1950-6929

Éditeur

LIT Verlag

\section{Édition imprimée}

Date de publication : 1 décembre 2002

\section{Référence électronique}

Fatoumata Ouattara, "Gouvernances quotidiennes au cœur des structures de santé. Les cas d'Orodara et de Banfora, Burkina Faso », Bulletin de l'APAD [En ligne], 23-24 | 2002, mis en ligne le 15 décembre 2006, consulté le 07 septembre 2020. URL : http://journals.openedition.org/apad/145 ; DOI : https://doi.org/10.4000/apad.145

Ce document a été généré automatiquement le 7 septembre 2020

Bulletin de I'APAD 


\title{
Gouvernances quotidiennes au cœur des structures de santé. Les cas d'Orodara et de Banfora, Burkina
}

\section{Faso}

\author{
Fatoumata Ouattara
}

\begin{abstract}
"C'est Dieu qui choisit le travail pour les gens, mais Dieu ne donne pas le cœur aux gens pour embrasser ces métiers. On dit que l'appétit vient en mangeant, il y a certains qui en mangeant ont l'appétit, il y a en aussi qui mangent mais quand ils refusent, ils refusent. (...) Dans les ENSP (Ecole Nationale de Santé Publique) au niveau du Burkina, il n'y a plus un contrôle, les gens sont pris au hasard, chacun veut avoir tout en un temps record. Ça, ça pousse les gens à faire ce qu'il ne faut pas faire." (un agent de santé au CHR de Banfora)
\end{abstract}

1 Dans de nombreux pays africains, les centres publics de soins se révèlent de plus en plus comme des lieux où la carence du service public est notoire et consternante : la "corruption-racket" s'y développe et devient systématique ${ }^{1}$. De telles pratiques sont tellement généralisées qu'elles semblent ne même plus émouvoir. Si l'on considère la santé comme un bien, on peut alors s'interroger sur les modalités des "gouvernances" (gestion des biens et des ressources) qui agissent au niveau du secteur public de la santé.

2 La carence de la gestion de la santé comme bien public conduit à une interrogation socio-anthropologique relative aux modalités de fonctionnement quotidiennes au sein des services locaux de santé publique, ainsi qu'aux justifications inférées (implicites ou explicites) tant par les agents de l'administration étatique que par les usagers ${ }^{2}$. La démarche qui guide cette recherche est d'appréhender les pratiques des acteurs en matière de santé à la croisée d'autres univers que celui de l'éthique professionnelle bio-médicale ${ }^{3}$.

3 S'agissant de la santé, la gouvernance peut aussi être envisagée du point de vue strictement individuel. De ce fait, dans une première partie, je m'attacherai à décrire les motivations du choix professionnel, les avantages que les professionnels associent 
au métier de soignant, les stratégies de gestion des carrières ainsi que les perceptions de la faute professionnelle. Sous l'angle institutionnel, la gouvernance met également en évidence les modalités de la gestion administrative des structures de santé. La deuxième partie de mon propos s'attachera à illustrer cette dimension pragmatique de la notion de gouvernance.

Les motivations du choix de la profession et les avantages du métier

4 Le choix de la profession apparaît comme une dimension déterminante pour comprendre la logique des conduites professionnelles et plus particulièrement des agents de santé (Rogoff 1957). Les professions médicales et para médicales restent de celles où on associe encore - ne serait-ce que de façon implicite - la vocation au choix de son exercice. Cependant, certains soignants du CMA d'Orodara reconnaissent être arrivés dans la médecine par le simple hasard des choses ou par un concours de circonstances à un moment de leur parcours scolaire. De ce fait, un rêve de jeune écolier ou collégien d'être un jour mécanicien ou pilote d'avion peut s'envoler quand une orientation arbitraire ${ }^{4}$ décide d'une spécialisation en médecine. La décision de passer le concours d'un métier médical trouve également sa justification dans un contexte de précarité de l'emploi et de crise économique accentué depuis une dizaine d'années. Il devient ainsi courant de rencontrer des anciens étudiants de géographie, de physique chimie, etc., dans des blouses d'infirmiers diplômés d'Etat.

$5 \quad$ L'exercice de la profession peut être dû à une confusion de dénomination. L'exemple le plus emblématique est celui de ce praticien qui décida de passer le concours de maïeuticien alors qu'il pensait exercer une profession en rapport avec la physique chimie, une matière scolaire qu'il affectionnait. La terminologie "maïeuticien" ${ }^{5}$ lui a fait penser à "mécanicien" plutôt qu'à un métier de la santé.

6 Si le hasard, le concours de circonstances, la confusion terminologique y sont pour quelque chose, d'autres praticiens de santé associent l'exercice de leur métier à un choix de longue date, effectué souvent depuis l'enfance. Un choix généralement consécutif à un événement de maladie personnelle ou d'un proche parent, expérience d'autant plus marquante quand la maladie conduit à la mort. Le choix de la médecine se définit alors comme un rêve d'enfance qui se cristallise sur les accessoires du métier : la blouse, le stéthoscope. Le paraître professionnel justifie alors le choix de la profession.

7 La fascination éprouvée à l'égard d'un soignant motiverait également le choix ou du moins l'admiration vis-à-vis du corps médical et para médical. On peut également avoir été impressionné à son jeune âge par la compétence technique d'un soignant et surtout par la reconnaissance sociale à l'égard du statut de soignant.

Certaines études menées dans les sociétés occidentales et consacrées à l'analyse du choix de la profession médicale (Becker 1962 ; Memmi 1979 \& 1980) tendent à montrer que la notion de pourvoyance médicale ${ }^{6}$, l'influence du milieu familial et du réseau de sociabilité sont des variables déterminantes dans le choix précoce d'un métier de la santé. Outre l'attrait accordé à la notion de pourvoyance médicale (motivation intrinsèque), la reconnaissance sociale (motivation extrinsèque) associée au statut de soignant paraît un facteur décisif dans le choix de la profession de soignant (Déchamp-Le Roux 1994). Or, on observe que si la précocité du choix du métier existe chez certains soignants du CMA d'Orodara, l'intérêt accordé à la reconnaissance sociale est beaucoup mis en avant. Comme le souligne Isabelle Gobatto à propos d'un groupe de médecins burkinabè, "les règles d'acquisition du capital social sont séparées des critères d'exercice de la profession. Les compétences professionnelles objectivées dans 
la performance au quotidien, sont placées au second plan" (Gobatto 1999 : 59). C'est alors qu'il devient intéressant de se pencher sur les avantages du métier reconnus comme tels par les soignants eux-mêmes.

Du pouvoir de soigner les "parents"...

Un des avantages de la profession reconnu par de nombreux soignants s'associe au pouvoir et au privilège de sauver des vies humaines et plus particulièrement celles du réseau de parents et de connaissances proches, réseau couramment désigné par l'acronyme PACA (parents, amis, connaissances et alliés) dans le jargon des fonctionnaires burkinabè. Cet avantage relationnel s'accompagne d'un atout matériel : la gratuité des soins et des frais d'hospitalisation ${ }^{7}$ pour les parents très proches.

Le goût du "cadeau" : reconnaissance ou "pot-de-vin" ?

ester sa reconnaissance à l'égard du soignant est une pratique courante dans le contexte ouest-africain. Elle se manifeste avant tout par une parole de remerciement de l'ancien patient, qui est souvent suivie par un cadeau que le patient, ou l'ancien patient, offre à son soignant pour attester sa reconnaissance. Ce type de conduite est perçu par le personnel soignant comme un avantage du métier.

11 On compare volontiers les ethnies que l'on classe en fonction de la générosité des patients. Par exemple, les Peul et les Mossi manifesteraient plus d'égards, voire plus de reconnaissance à l'égard des personnels soignants que les Sénoufo.

La générosité des patients apparaît donc comme une caractéristique importante dans l'élaboration des catégories culturelles construites par les soignants. D'un point de vue extérieur, qu'un patient offre un présent à celui qui l'a soigné n'a rien de surprenant. Car il s'agit avant tout de la manifestation d'une reconnaissance en accord avec le code de bienséance locale. On est donc ici dans le cadre de la norme sociale. Cependant, bien souvent, le don semble être suscité par le soignant, tout au moins implicitement. C'est alors que la frontière entre le cadeau (le don d'un bien dénué de contrainte) et la corruption (le don suscité, contraint par un intérêt) peut paraitre floue ${ }^{8}$. Mais on reviendra sur ce point. Pour l'instant, voyons les autres avantages imputés à la pratique médicale.

Le goût de gouverner...

13 L'organisation, la responsabilité, le pouvoir de décision, voire l'autorité exercée dans une position de pouvoir sur les pairs sont autant d'éléments qui apparaissent aux soignants d'Orodara comme des avantages liés à leur pratique professionnelle. Les gens vont jusqu'à présenter le goût du pouvoir comme une motivation dans le choix de ce métier. Ainsi une sage-femme d'Etat précisait que si elle avait été impressionnée par la blouse rose des sages-femmes, ce qui lui a surtout plu, c'était l'autorité que les sages-femmes avaient sur les accoucheuses. Elle s'empressait de souligner cependant que la compétence technique était quand même une condition sine qua non à l'exercice efficace de ce pouvoir.

Mais il n'y a pas que des avantages. En effet, bien qu'ils soient régulièrement payés, les soignants soulignent l'insuffisance de leurs salaires. Ils précisent (explicitement ou implicitement) la nécessité d'avoir des activités parallèles au métier médical afin d'avoir des revenus complémentaires.

La pluri-activité

15 L'exercice d'une autre activité éloignée tant de la profession médicale que du lieu d'exercice de la profession est banal chez les personnels de santé. Par exemple, il est 
très courant que les hommes possèdent des champs de culture, des vergers et que les femmes soient impliquées dans des activités commerciales. Mais la pluri-activité induit des confusions d'espaces et des interférences. Certains soignants considèrent en effet leur lieu de travail comme une enceinte privée au sein de laquelle ils disposent d'un privilège pour vendre des sandwichs et des boissons. De ce fait, une employée du CMA ne verra pas d'inconvénient à empêcher une commerçante de venir vendre ses sandwichs dans l'enceinte du CMA afin qu'elle ne lui fasse pas concurrence. Il lui semble tout à fait normal d'alterner ses activités de soignante avec celle de vendeuse de sandwichs à ses collègues. Les soignants conjuguent ainsi une activité médicale avec une autre occupation à laquelle on consacre souvent plus de temps de travail.

Mais ils peuvent aussi exercer des activités relatives à la profession à l'extérieur de la structure médicale. C'est l'exemple de ce manœuvre qui pratique la circoncision et d'autres actes de petite chirurgie en dehors de la structure de soin, contre rémunération. Cependant, c'est à l'intérieur de la structure médicale que certains actes sont rémunérés tant et si bien que l'on est tenté de parler de corruption. Prenons un exemple de situation entre un médecin généraliste et des patients.

Deux hommes entrent dans le bureau du médecin généraliste. Le patient remet les résultats d'analyse au médecin. Celui-ci regarde attentivement les papiers, il secoue la tête, regarde le plafond. Le malade a l'air inquiet. Il fixe le médecin du regard. Mais le médecin le rassure : " mon ami, calme-toi. N'aies pas peur. Pourquoi as-tu peur ? Dieu est grand, ça va s'arranger grâce à Dieu. Dieu est puissant (II regarde encore les résultats, et poursuit). N'aies pas peur. Tu n'es pas tranquille. En tout cas, tu n'as pas le sida. Tu as un vers un peu difficile, mais s'il plaît à Dieu, on va te soigner, tu vas guérir. Bon tu vas sortir, je vais voir l'autre (il parle de l'accompagnant) et ensuite tu vas rentrer". (Le malade sort). Le médecin à l'accompagnant : "on peut se voir ici ou bien c'est entre nous seulement ?" L'accompagnant : "entre nous deux." Les deux hommes se lèvent et entrent dans la pièce d'auscultation. Mais avant de rentrer, le médecin appelle un IDE à qui il demande de lui acheter une cigarette. Dix minutes plus tard, l'IDE revient avec la cigarette, il tape à la porte. Le médecin ouvre, les deux hommes s'apprêtent à sortir. Le médecin tient dans la main un billet de $5000 \mathrm{~F}$. Il me présente son cadeau : "Ah! vous voyez, je suis vraiment comblé, il vient de me faire un cadeau." Il ouvre la porte du bureau et présente le billet aux patients qui attendent dans la salle d'attente. "Vraiment, il faut le remercier, il vient de me faire un cadeau. Remerciez-le pour moi." Les patients remercient le bienfaiteur, ils font des bénédictions.

Le médecin regagne son fauteuil; il empoche le billet et précise qu'il y a un jour où il est rentré à la maison avec $60000 \mathrm{~F}$ CFA.

Bien qu'extrême, la situation décrite ci-dessus montre clairement l'enchevêtrement entre diverses normes ${ }^{9}$ : bienséance locale (reconnaissance, remerciement) et déontologie de soin (discrétion). Ce type de relations entre un professionnel de santé et ses patients nous amène à réfléchir sur les frontières symboliques de certaines pratiques sociales qui sont au cœur des interactions entre soignants et soignés. En particulier, on peut se demander où s'arrête le cadeau en tant que marque de reconnaissance et où commence le pot-de-vin en tant que pratique de corruption. Car le cadeau caractérisé comme "gratification" s'apparente bien souvent à une corruption. Mais ces cadeaux offerts souvent par les patients sont justifiés par les agents de santé comme une simple manifestation de reconnaissance. 
20 À bien des égards, en effet, offrir "quelque chose" au soignant - peu importe le moment - conduit in fine à la pérennisation de la relation clientéliste (Olivier de Sardan 2001). Le cadeau intervient donc dans la constitution d'un capital relationnel. La spécificité de l'interaction soignants/soignés semble jouer un rôle important par l'urgence et l'enjeu de l'acte médical : il y est question de la vie humaine. Ce type de pratiques fonctionne parce qu'il s'appuie sur un fond commun de représentations partagées entre soignants et soignés ${ }^{10}$.

Les reventes à titre privé de médicaments et la "gestion" de certains patients

21 Les reventes à titre privé de médicaments et le détournement du matériel à des fins privées sont également courantes au sein du centre médical. Le discours bien connu des partenaires de l'aide au développement, sur le manque de matériel médical et de médicaments se conjugue à des pratiques systématiques de détournement des dotations en médicament et en matériel.

Les usagers victimes de ce type de pratiques sont de fait les plus vulnérables. Cette vulnérabilité est généralement rendue par une dimension sociale et/ou économique accentuée par l'urgence. Les maternités urbaines représentent des services où les "deals" permettent à de nombreux agents de santé de se faire un surplus financier. Outre l'urgence de l'intervention médicale, en raison de la discrétion qui entoure l'évocation du sexe et de la sexualité, il est courant qu'un agent utilise cette discrétion pour faire une proposition de vente de médicaments à son patient. On est donc en présence de la surexploitation d'une notion sociale (discrétion) dans un univers professionnel où les agents de santé gouvernent. Une situation observée dans un centre de santé du sud-ouest du Burkina Faso peut nous servir d'exemple :

Il est $19 \mathrm{~h}$ et un infirmier de garde reçoit un patient qui se plaint d'une maladie dont les symptômes se situent au niveau du sexe.

-J'urine, ça brûle, quand je conduis (rapport sexuel), ça broie, je m'étais fait piquer mais ça recommence.

-Est-ce que tu as fait ce qu'on t'a dit ?

-Oui, j'ai fait, depuis 14 mois, je n'ai pas d'autres rapports avec une autre femme que mon épouse. (...) Maintenant, c'est comme une plaie, c'est pour ça que je reviens pour qu'on me pique.

(...)

-Tu n'as pas de copine?

-Non.

Après un interrogatoire, l'infirmier suggère au patient : "à ton retour, tu reviens me voir. Moi, je ne suis pas ici, ... même si c'est la nuit, viens me voir pour que je voie comment ça se passe".

Le major entre et reconnaît le patient.

L'infirmier rédige une longue liste de médicaments à acheter. Mais au lieu de remettre l'ordonnance au patient, il décide de l'aider. Il prend la calculatrice et déclare au bout de quelques instants : "si tu veux acheter tout ça, ça va te coûter 15 mille et quelques. Moi je peux t'aider, on est entre nous. Ça va te coûter 8300." Le patient paye.

D'autre part, outre cette dimension sociale, les personnes infectées par le VIH/Sida ou soupçonnées de l'être, sont souvent présentées par les agents de santé comme étant des "proies faciles" pour la revente de médicaments. Il s'agit en effet de personnes vulnérables d'un point de vue économique en raison de leurs hospitalisations multiples. Il y a là un paradoxe car la gratuité des médicaments destinés gratuitement aux PVVIH ${ }^{11}$ est problématique en raison des difficultés sociales consécutives à l'annonce de la 
séropositivité. Quoi qu'il en soit, la revente des produits aux patients est ce que de nombreux soignants appellent "le marché noir" ou le deal.

Pour la majorité des agents de santé rencontrés, le détournement des biens appartenant au service représente un complément de ressources. Qu'un chauffeur d'ambulance fasse pression sur les parents d'un malade (dont l'état de santé requiert un transport urgent vers le $\mathrm{CHN}{ }^{12}$ de Bobo-Dioulasso) pour payer plus de carburant qu'il n'est nécessaire pour le trajet n'étonne personne dans le service.

L'appartenance à certains organes de gestion sanitaire

L'appartenance à certains organismes de gestion sanitaire peut être également un moyen de légitimation des pratiques illicites conduites dans le centre médical. Etre membre de l'ECD ${ }^{13}$ et/ou du comité de gestion sont autant de positions stratégiques permettant le détournement des ressources allouées à la gestion du CMA ou du district. Et l'appartenance à cet organe, conçu au départ comme un cadre de réflexion et d'organisation du système sanitaire local, semble plus guidée par le privilège de participer, au réseau de clientèle des dirigeants que sur un critère de compétence. A ce titre, n'importe qui ne fait pas partie de l'ECD. Un membre de cet organe le compare à un gouvernement ${ }^{14}$ :

"Je fais partie de l'ECD, l'ECD est le moteur du district, c'est un rôle de coordination, c'est comme un gouvernement en fait. C'est comme si le district était un pays, dans l'ECD chaque ministre a un rôle défini. Je suis responsable des activités de suivi et d'évaluation. Il y a la supervision, les contrôles, les monitoring. C'est simplement voir une fois par trimestre ce qu'il faut faire au niveau des activités."

Qu'en est-il du Coges ? Préalablement défini comme un organe structurant l'implication de la communauté locale dans la gestion des affaires de santé (initiée par la politique de recouvrement des coûts), le Coges a fini par constituer pour les soignants une sorte de paravent pour dissimuler les détournements de fonds publics destinés à la prise en charge de la santé communautaire. Pourtant certains responsables s'acharnent toujours à définir le bien fondé de cette structure.

A l'image de nombreux Coges, outre des représentants de la collectivité locale, celui d'Orodara comprend quelques personnels du centre médical. Ainsi, le major du CMA occupe la fonction de conseiller technique et le gestionnaire du CMA tient les rênes de la gestion du comité. En définitive, le Coges reste pour de nombreux soignants, un organe géré par le MCD qui finit par en faire une source personnelle de revenus complémentaires. Un employé du centre explique ainsi calmement comment les détournements au sein du Coges ont permis à un MCD de se payer des biens luxueux. La présentation des vœux constitue une occasion souvent idéale pour les membres du bureau du Coges pour pouvoir faire des détournements de fonds de la structure. Aussi, entend-on dire souvent en plein mois de mars que les personnels d'un établissement de santé procèdent à la présentation des vœux de nouvelle année à leurs responsables.

La gestion de projets de développement

La gestion d'un projet est souvent l'opportunité d'une source de revenus complémentaires au salaire car la responsabilité d'un projet n'est pas sans lien avec le statut que l'on occupe dans la hiérarchie du centre de soin. Cela n'étonnera donc guère d'apprendre que la sage-femme responsable du projet de planification familiale est la compagne du MCD ou que celui-ci soit lui-même responsable d'un ou de plusieurs projets de santé publique. Une sage-femme a pu d'ailleurs m'expliquer, non sans fierté, comment elle a reçu deux fois de suite un financement de deux millions cinq cent mille 
francs CFA dans le cadre d'un projet destiné à la formation d'accoucheuses villageoises. En plus de ce projet et de son salaire de sage-femme, elle précise recevoir une somme de $30000 \mathrm{~F}$ CFA par mois, pour la gestion d'un projet de sensibilisation des femmes à l'utilisation des contraceptifs. On comprend donc son inquiétude suite à l'affectation récente de son compagnon dans une grande ville. Car bien que ce changement de poste soit une promotion professionnelle pour celui-ci, ce ne sera pas le cas pour la compagne, qui risque de perdre les avantages matériels dont elle bénéficiait à Orodara du fait de l'absence de concurrence par rapport à ses pairs et, surtout, de son statut de compagne de MCD. La gestion conjointe de deux projets lui permet ainsi d'augmenter les ressources financières.

Comme cela se présente pour les autres organes décisionnels, n'importe qui ne gère pas les projets : "il faut avoir une place de décision pour écrire des projets". Mais en ville, compte tenu du nombre important de soignants, la compétition pour la gestion des projets est plus âpre. En outre, les postes de responsabilité sont comptés.

31 Les stratégies pour bénéficier des formations en santé publique ou pour être responsable de projets s'élaborent dans une perspective d'augmentation du revenu personnel. Mais l'accès à de telles ressources est lié à la position occupée et à l'entregent dont on dispose au sein de la hiérarchie institutionnelle et politique ${ }^{15}$. En outre, la compétition pour de tels postes devient très âpre dans le contexte urbain où les demandes de formation et de postes de gestion de projets sont plus nombreuses. En dehors des affectations arbitraires, le choix du lieu d'affectation répond souvent à des contraintes d'ordre familial (désir de se rapprocher de la famille, une mutation demandée pour être auprès d'un enfant en âge d'aller au lycée, une demande d'affectation en raison des avantages économiques de la localité demandée, etc.). La comparaison entre la ville et la campagne est récurrente. Les personnels de santé mettent souvent en balance les avantages en nature attachés aux affectations en milieu rural avec les "deals" financiers qu'offre l'activité professionnelle dans les grandes villes. Un IDE se réfère ainsi à ce qu'un de ses supérieurs hiérarchiques avait lancé publiquement pour expliquer son goût des perdiem et de leur montant :

"... quand on l'appelle pour une formation, il demande d'abord le montant du perdiem. Il dit qu'une prise en charge journalière inférieure à $10000 \mathrm{~F}$ il ne prend pas. Tout ça fait qu'à l'hôpital, on rançonne les malades. Les soignants sont à la chasse des malades pour les rançonner."

Le dysfonctionnement des structures publiques de soins, caractérisé entre autres par l'inertie de nombreux soignants et les pratiques de racket, est souvent expliqué par les agents de santé comme étant la conséquence d'une situation de déséquilibre entre la santé publique et la santé clinique. Celle-ci serait moins bien rémunérée que celle-là. Ces écarts au niveau de la rémunération sont d'autant plus difficilement admis qu'ils sont inversement proportionnels à la durée des études. Alors que la compétence requise pour être praticien de santé clinique nécessite plusieurs années d'études, la formation en santé publique ne requiert que 9 mois d'études. Face à de telles réalités, les professionnels de santé affectés dans des structures de soins publics urbaines ne semblent pas avoir d'autre choix que de mettre en œuvre des pratiques de "racket" pour arrondir les fins de mois et faire face aux nombreuses pressions sociales. Dans les grandes villes, ces pratiques quotidiennes sont telles que certains soignants comparent la cour de l'hôpital à un terrain de chasse où chacun (soignant) se lance à la recherche d'une proie. 
33 En somme, la pluri-activité à laquelle se livrent les fonctionnaires de l'Etat conduit à une quasi confusion entre la sphère publique et la sphère privée, une sorte de "porosité" qui conduit in fine à "un écart maximum entre normes officielles et normes pratiques" (Chauveau et al., 2001). Toutefois, on peut considérer les points sur lesquels se fonde le consensus entre les personnels soignants et les points de désaccords, autrement dit les sources de conflits.

Consensus et désaccords sur ce qui doit se faire

L'observation des conduites quotidiennes donne à penser qu'il existe un certain nombre de points auxquels la majorité des soignants adhère. Ainsi, la banalité des retards s'observe ici et là. Dans l'interaction avec les patients, on note une certaine unanimité sur une sorte de "tri" implicite de ceux-ci. Autrement dit, il y a ceux à qui l'on accorde une priorité dans les soins et les autres qui ne nécessitent pas plus d'attention que cela. Bien entendu, la mobilisation pour le réseau de "parents, amis, connaissance et alliés" (PACA) est indiscutable. Ainsi, les patients qui ont de l'argent ou qui sont suspectés d'en avoir passeront avant les autres.

Cependant, on ne s'inquiètera pas d'abandonner un enfant dans le coma dont les parents originaires d'un village voisin sont manifestement démunis aussi bien d'un point de vue financier que relationnel (tant à l'hôpital qu'en ville). Ce jeune malade ne suscitera qu'un minimum d'attention jusqu'à ce que la gravité de son état finisse par mettre timidement les soignants sur la voie d'un transfert vers l'hôpital urbain de Bobo-Dioulasso.

36 Autrement dit, sans connaissance et sans argent, on risque fort pour sa vie ${ }^{16}$. Outre la variation de la mobilisation des soignants pour les patients en fonction de critères économiques et sociaux, une norme semble dominante dans les pratiques observées. En effet, on remarquera que les décisions de références vers un plateau technique supérieur tardent souvent en dépit de l'état d'urgence. La référence est rapidement faite dès que le diagnostic est clairement établi. Cependant elle tardera s'il se fait un doute sur le diagnostic, car les soignants craignent que leurs collègues de ville n'établissent une incompétence s'ils réalisent que la pathologie référée aurait pu bien être prise en charge dans la structure de référence. Dans ce cas, tous les bricolages et tâtonnements sont permis pour éviter de perdre la face. De façon paradoxale, bien qu'il s'agisse d'une question de vie ou de mort pour le patient, c'est le risque de honte pour le soignant qui se trouve ainsi être mis en avant. On est donc dans une logique où le traitement social prévaut sur le traitement médical : il faut montrer son savoir soigner pour éviter la honte. La récurrence de certaines techniques médicales peut susciter des interrogations chez n'importe quel non praticien: la ponction $d u$ liquide céphalo-rachidien, la ponction du liquide ascitique, le placement de la perfusion. Même si dans certains cas, ces actes ne sont pas sans lien avec une demande insistante du malade, les soignants les associent souvent à une preuve de compétence ou tout simplement à la pratique de leur métier. A cet égard, il serait intéressant d'observer les pratiques en cours au sein des formations pour analyser plus en profondeur la fréquence de tels actes chez les praticiens de santé.

37 En revanche, d'autres pratiques ne font guère l'unanimité chez les acteurs de soins. Les désaccords se font d'abord sur des questions d'organisation du travail et sur le fonctionnement interne de la structure. La rareté sinon l'inexistence de visite médicale dans un CMA, malgré la présence d'un médecin généraliste pendant la période de l'enquête, n'est guère tolérée par les agents de santé - à tout le moins dans les discours. 
Les infirmiers font souvent remarquer que l'absence de visite médicale en présence du médecin limite le transfert de connaissances. Les rares fois où elles ont lieu, ces visites sont des situations où le médecin s'adresse à ses collègues infirmiers dans un jargon qui leur est difficilement accessible. En outre, le médecin semble peu ouvert à toute interrogation qui lui parait comme une remise en question de ses connaissances dans le domaine médical. Outre les conflits de compétence que l'on peut retrouver au cœur de n'importe quel groupe professionnel, il y a ici la légitimité d'un médecin généraliste qui pose problème au sein d'une structure de santé. Il s'agit donc là d'une situation de crise de fonctionnement qui ne peut être extrapolée aux mécanismes généraux des structures de santé.

Certaines pratiques très courantes semblent largement acceptées bien que non recommandées par la déontologie médicale : le retard au service, une certaine tendance à privilégier les patients en fonction de la distance sociale et/ou de la richesse apparente. A cet égard, ceux qui en en font trop, tout comme ceux qui n'en font pas assez, sont considérés comme des marginaux et sont sanctionnés comme tels. Selon Jean-Pierre Olivier de Sardan (2001), ces logiques partagées par les agents de services de la fonction publique dans les plusieurs pays ouest-africains, seraient induites par la combinaison de "cultures professionnelles locales" s'associant aux normes pratiques du métier et d'une "culture bureaucratique privatisée" alliant "privilégisme, privatisation interne, clientélisme, improductivité, déshumanisation et sur-personnalisation". Une culture professionnelle dans les systèmes de santé ouest-africains. Tout au moins, ce déséquilibre entre la survalorisation $d u$ domaine social d'une part et la sous-valorisation du domaine professionnel semble parfois analysé comme un mode de résolution des tensions induites par le décalage entre illusions et attentes du métier et la réalité à laquelle les soignants sont confrontés dans le quotidien de leur exercice professionnel (Gobatto op. cit. : 59). Mais bien plus qu'une confrontation d'univers de référence, les acteurs sociaux se meuvent dans un "pluralisme normatif'. Cette pluralité de normes dont on a affaire dans le cadre du service public tend à rendre informelle la "politique publique" et légitime, par voie de conséquence, la production des réseaux clientélistes (Chauveau et al. $2001: 152$ ).

À bien des égards, dans les situations d'interaction avec les usagers de ce centre public de santé, tout laisse supposer qu'il y a d'un côté ceux qui bénéficient d'un certain nombre de privilèges et de l'autre, ceux qui sont "obligés" de donner quelque chose en échange d'un service public. On paie pour ne plus être cantonné dans les difficultés des services de la santé et ne plus subir les conséquences de l'anonymat. Cette inégalité d'accès au service public de la santé reste souvent légitimée par les usagers eux-mêmes. Il faut user de son réseau de connaissance ou payer pour accéder au service des agents de la santé. Clientélisme et corruption trouvent ici leurs justifications. Selon Jean-François Médard, le clientélisme en tant que "relation d'échange social entre inégaux" implique particularisme et favoritisme; tandis que la corruption à travers l'échange économique qu'elle sous-tend remet en question les principes de l'universalisme et de l'égalité qui fondent la notion même de service public (Médard 2000 : 84). Si de tels phénomènes trouvent place là où le fonctionnement de l'Etat ressort plutôt du néopatrimonialisme (Médard 1995 \& 1998), la dynamique des liens de parenté et des réseaux de sociabilité semble les légitimer (Bouju 2000). Si on soigne un parent, il ne devrait pas oublier, en retour, de faire les salutations et un cadeau. La relation de soins est aussi une occasion pour réactiver un lien d'amitié. Mais quand on n'a ni parent, ni ami, ni connaissance, on s'arrange pour en créer. Si dispensaires et 
hôpitaux sont des lieux de sociabilité comme toute sphère de relations sociales, cette sociabilité reste une dimension associée à la qualité de l'échange social. Autrement dit, les "mains vides" ne font ni la parenté, ni l'amitié, ni la santé. D'une certaine manière, que l'on soit "parent", "ami" ou anonyme, la procuration de soin engage l'individu dans une situation "d'endettement" à l'égard du soignant. Les anonymes payent évidemment le prix fort puisqu'ils sont pratiquement contraints de monnayer dans l'urgence le service. Mais le "parent" qui a bénéficié des faveurs sait aussi que tôt ou tard, il devra payer cette faveur sous une quelconque forme. En effet, l'interconnaissance qui caractérise les relations sociales en milieu rural et dans les petits centres urbains ne facilite pas le monnayage direct des actes médicaux, on est là plutôt dans la sphère du cadeau en tant que manifestation de reconnaissance du lien social. Il ne s'agit pas ici de prétendre que les pratiques de corruption seraient inexistantes en milieu rural. Loin de là ! Les pratiques illicites y prennent tout simplement d'autres formes, sans doute moins criantes, que celles que l'on peut observer dans les grandes villes où le degré d'interconnaissance est moins élevé.

En ce qui concerne les rapports de génération, outre l'amélioration de l'expérience médicale proprement dite, l'apprentissage des "jeunes" auprès des "aînés" se fait souvent à un autre niveau: l'apprentissage de certaines pratiques conjointes au métier, telle les techniques de revente du petit matériel médical. Mais seule la reconnaissance de la compétence professionnelle d'un aîné préside à un tel apprentissage. Dans un service, après s'être tenu comme un "élève de l'école coranique" auprès du responsable de son service, un jeune IDE a fini par recevoir de ce dernier l'information qui consiste à savoir comment revendre aux patients des poches de recueil de liquide ascitique. Il n'est guère surprenant qu'une telle pratique puisse encourager la fréquence de certains examens médicaux.

40 Certaines règles de conduites sont donc requises pour conquérir la confiance des aînés et pour établir du même coup l'intégration des "cadets" au sein du groupe : faire les consultations à la place de l'aîné, le remplacer pour une garde s'il en fait la demande. Le respect de l'aîné se fait même souvent au détriment de la santé du patient. Par exemple, le jeune évitera de lui dire que le diagnostic qu'il vient d'établir est inexact même s'il en a la preuve flagrante. Dans le meilleur des cas, il prescrira un autre traitement en toute discrétion au patient en cas d'hospitalisation, comme me l'a confié un jeune soignant. Mais les relations entre "anciens" et "nouveaux" ne se font pas toujours sous le sceau de la soumission.

41 En revanche, des soignants nouvellement recrutés ne manquent pas de regard critique sur les conduites professionnelles de ceux qu'ils appellent ironiquement les "vieux". Ceux-ci sont décrits comme des gens désabusés qui transgressent les règles de base de la pratique médicale. Par exemple, ils ne tiendraient pas compte du bon accueil qui devrait être réservé aux patients pour atténuer leur appréhension à l'égard de l'autorité médicale mais également de leur douleur, à tout le moins d'un point de vue psychologique. En outre, le suivi minimum des patients ne serait pas respecté.

42 Toutefois, deux tendances au niveau des conduites des soignants tendent à être mises en évidence : il y a d'un côté ceux qui n'en font pas assez et de l'autre ceux qui en font trop. À côté de ceux qui ne manquent pas de regard critique sur les conduites de leurs collègues mais qui en tout état de cause semblent intégrés au système de fonctionnement de la structure, on observe une catégorie de soignants que l'on pourrait, à défaut d'une autre terminologie, qualifier de marginaux. Ils semblent se situer à la marge du système de fonctionnement en cours. Un premier groupe serait constitué de soignants dont les pratiques débordent largement d'un point de vue 
négatif, des règles admises dans le groupe. Deux soignants reconnus par leurs collègues comme des alcooliques appartiennent à ce groupe de marginaux-type. Tous les deux passent une grande part de leur temps dans les bars et buvettes et n'épargnent pas le lieu de travail de leur état d'ivresse : retard abusif au niveau des horaires, abandon du poste de garde pour consommer de la bière de mil, refus de soigner certains patients en raison d'un état d'inconscience, voilà autant de critères qui disqualifient ces soignants aux yeux de leurs collègues.

D'un autre côté, une catégorie de soignants - bien que loin d'appartenir à la précédente - peut être considérée comme étant marginale dans la mesure où les conduites de ces soignants ressortissent à une a-normalité par rapport aux normes en cours. Par exemple, ce maïeuticien en poste à Orodara depuis quelques années, dont la réputation non seulement de la compétence technique mais aussi de la conscience professionnelle auprès de ses pairs et auprès de la population est sans commune mesure. Ainsi, la population n'a pas hésité à manifester devant le haut-commissariat de la ville, une fois que la nouvelle sur la décision d'affectation du jeune soignant a circulé dans toutes les familles. Cette affectation a d'ailleurs fini par être annulée tant l'opposition des habitants d'Orodara était manifeste au départ de ce maïeuticien irréprochable. En effet, la particularité de la conduite de ce soignant est frappante. Contrairement à la majorité de ses collègues, il est ponctuel au service; il ne semble pas ménager ses efforts pour prêter une écoute attentive aux patientes. Ses collègues le raillent même à ce propos. Les tournées de vaccinations dans les villages semblent ne lui poser guère de soucis. Son omniprésence à la maternité est notoire.

Même après avoir subi une perfusion à domicile, il n'hésite pas à se rendre à son lieu de travail pour y effectuer d'éventuelles tâches. Ce soignant qui semble être guidé par une conscience et une passion pour son travail fini par paraitre atypique aux yeux de ses collègues.

\section{Gouvernances personnalisées}

$\mathrm{Au}$ niveau de la gouvernance administrative locale, chaque nouveau responsable adopte un mode de conduite particulier. Nous sommes en présence de modes de gestion personnalisés et diversifiés des ressources associées à la santé. Voyons par exemple, comment un IDE institue sa "gouvernance" au sein d'un CSPS où il vient d'être affecté.

"Quand je suis arrivé, ils ont voulu que j'adopte les méthodes de mes prédécesseurs qui aimaient administrer des soins à domicile. Très rapidement, j'ai convoqué les notables pour leur donner mes méthodes de travail: je viens le matin, s'il y a un malade, je le traite, un autre arrive, je le traite. Maintenant que ce soit moi qui vienne attendre le malade, ce n'est pas moi. Parce que j'ai d'autres choses à faire et je suis humain, j'ai d'autres besoins à satisfaire. C'est dans la fourchette de $7 \mathrm{~h} 30$ à 10 heures, après 10 heures, tu viens me chercher au village. Moi en sortant, je dis à ma femme que si quelqu'un arrive il faut aller me voir dans tel coin. A partir de 10 $\mathrm{h}$, moi je quitte mon poste. Je vais prendre un pot. Si on a besoin de moi, si la vie d'un être humain est en danger, on vient me chercher dans un coin. A chaque fois qu'on vient me chercher, j'enjambe ma calebasse (bière de mil). Je viens faire ce que je peux et si je peux repartir, je repars (...). Je suis buveur, mais je condamne les buveurs. Je suis parfois d'accord avec les musulmans qui veulent interdire le dolo, je les comprends dans la mesure où il faut interdire l'alcool à ceux qui n'arrivent pas à se contrôler. Même en médecine, si tu ne supportes pas un produit, il faut interdire l'administration de ce produit. Moi, je suis convaincu que même l'imam peut boire sans problème s'il est lucide. Un agent de santé qui peinte (consomme de l'alcool) et qui n'arrive à administrer les soins est bon à fusiller. Cela veut dire que même s'il n'avait pas bu, c'est un criminel. Le fait de boire ne peut pas jouer sur la 
compétence. En principe, c'est une question de personnalité, quand tu es compétent, l'alcool ne peut pas altérer cette compétence. Il y a des gens qui n'ont pas bu grand chose et qui font n'importe quoi, il yen a d'autres qui boivent et qui sont corrects." (IDE)

À la suite de ce qui vient d'être décrit, il apparaît que dimension économique et politique s'imbriquent étroitement dans les gouvernances personnalisées. L'éthique professionnelle des agents de santé conjugue une diversité de gouvernances : économique, sociale, politique, religieuse. Ces dimensions s'imbriquent étroitement et structurent de facto la gestion de l'espace public de la santé.

Le cadre institutionnel des services de santé se caractérise ainsi par une inadéquation entre les textes de lois en matière de gestion administrative et leur mise en application.

Le cas exemplaire est celui du Centre Hospitalier de Banfora, où un surendettement financier chronique fait l'objet d'une crise rapportée dans la presse nationale. En dépit d'un déficit budgétaire annoncé, le directeur a continué à ordonner les sorties de caisses. Cette conduite administrative a été récompensée par la nomination de l'auteur de la faute à un poste de haute responsabilité. La nomination apparaît ainsi comme "une prime à la mauvaise gestion". C'est le même administrateur hospitalier, alors qu'il était directeur des services économiques du CHN de Bobo-Dioulasso, qui avait procédé à l'ordonnance d'une commande fictive de matériel bio-médical s'élevant à $76580000 \mathrm{~F}$ CFA. La mise à jour de cette manœuvre de fausse facture avait conduit à sa nomination comme Directeur Général du CHR de Banfora (ville d'origine du ministre de la santé). L'enquête alors annoncée à la suite de cette affaire a été classée. La crise du déficit budgétaire au sein de l'hôpital de Banfora conduira encore à une ascension professionnelle du DG, puisqu'il sera appelé à Ouagadougou pour un poste de haute responsabilité. En dépit de sa responsabilité mise à jour dans la situation déficitaire de la structure hospitalière, l'affectation du DG sera désapprouvée d'une part par certains de ses collègues et d'autre part par les notables de la ville. En effet, la présidente de la Commission des Affaires Générales et Institutionnelles (CAGI) originaire de la ville, ne cache pas son désaccord face à la mutation du DG, qu'elle qualifie d'être "sociable". Par ailleurs, les discours de regret des notables seront explicites à l'occasion de la cérémonie de passation de service entre l'ancien et le nouveau responsable du CHR. Le message d'un griot qui s'adresse au Haut-Commissaire est sans équivoque :

"Ceux qui sont ici rassemblés te demandent de dire à T. L. (alors ministre et originaire de la ville), de dire à M. (un élu de l'Assemblée Nationale) et qu'à son tour lui aussi dise à Blaise Compaoré, que nous sommes extrêmement mécontents du départ de T. A." (L'Evénement, $\mathrm{n}^{\circ} 10: 10$ ).

Le représentant de la communauté musulmane ne cache pas lui non plus son jugement sur le partant :

"T. A. est un homme très gentil et bien éduqué ; mais ce qu'il faut savoir c'est que si T. A. est si bien, c'est parce qu'il a eu de bons amis comme O. M. P. (responsable d'une société de nettoyage qui avait le monopole sur l'entretien des locaux du CHR) notre fils que je salue au passage, grâce à qui nos femmes ont eu du boulot. Si tu suis des gens bien, tu seras un homme bien, si tu suis des gens mauvais tu ne peux être que mauvais" (L'Événement, $\left.\mathrm{n}^{\circ} 10: 10\right)$.

Un troisième intervenant, un responsable coutumier, sera plus modéré dans ses propos : "si nous avons prodigué des bénédictions à notre fils qui s'en va, faisons autant pour celui qui arrive parce que c'est aussi notre fils". 
51 Ce qu'il faut peut-être avoir présent à l'esprit, c'est que T.A. avait auparavant octroyé à une société locale de gardiennage un contrat mensuel d'un montant de 174640 F CFA et que ce contrat a été interrompu avec l'arrivée du nouveau directeur pour des raisons budgétaires. De même, le contrat de restauration qui était conclu avec une restauratrice de la ville a été réduit avant qu'elle décide de mettre fin au contrat pour une question d'insuffisance de commandes. Au lieu de livrer des repas au personnel de garde et aux malades, on ne lui demande que les repas pour le personnel de garde. Cette attribution de marché était liée à un réseau de clientèle. Aussi, on pouvait imaginer combien l'intégration, pour le nouveau DG, n'allait pas aller de soi, surtout s'il commençait par la clôture des marchés octroyés par son prédécesseur. On voit bien comment l'administration d'une structure, de santé implique des acteurs internes et externes. Il est question de positionnement par rapport à des réseaux d'intérêts.

52 L'instabilité des responsables s'associe à une instabilité des politiques de gestion et de fonctionnement des structures médicales. A chaque nouvelle affectation de responsable, on assiste à une nouvelle "gouvernance hospitalière" qui ne prend pas toujours en compte les acquis du prédécesseur. A chaque responsable, ses logiques d'organisation de la structure, sans tenir compte des acquis de la précédente organisation.

53 Les critères de nomination changent souvent d'un directeur à l'autre : par exemple, pour être nommé responsable d'une unité de soins (major), un responsable de structure institue les critères d'âge et d'ancienneté (10 ans minimum de service au sein du service).

54 Ainsi, les affectations internes du personnel sont fréquentes. Les mutations internes ne reposent pas forcément sur des critères d'expérience et de compétence. Par exemple, une infirmière jugée compétente par ses collègues et par les usagers sera nommée comme responsable du service de l'hygiène. Le directeur donnera les raisons de cette nomination :

"Je veux une infirmière pour la responsabiliser dans le domaine de l'hygiène. Quelqu'un qui peut faire le rôle d'infirmier hygiéniste à l'hôpital parce qu'en ce temps de Sida, la question de la collecte, du stockage et de l'évacuation des déchets hospitaliers est une question importante. (...) Elle s'occupe des brancardiers parce que la morgue pose aussi un problème d'hygiène." (L'Evènement, $\mathrm{n}^{\circ} 10: 10$ )

Quand un journaliste interroge l'ancien DG sur le bien fondé des accusations de mauvaise gestion dont il fait l'objet, sa réponse oscille entre le déni et la légitimation de son statut, qui lui conférait un pouvoir ne pouvant pas être remis en question : "En tant que principal ordonnateur, $j$ 'ai des pouvoirs. On ne peut pas me reprocher d'avoir exercé ces pouvoirs qui, d'ailleurs, se sont faits dans le cadre de la loi." Ce sur quoi ce responsable administratif insiste le plus est la conséquence sociale des accusations dont il fait l'objet : son image risque d'être ternie !

56 Ce qui vient d'être dit des pratiques médicales des agents de santé et des modes de directions administratives conduit à s'interroger sur les effets qu'entraînent de tels déséquilibres sur les prestations des soignants et sur la gestion publique de la santé des populations. A ce titre, le thème des perceptions des erreurs professionnelles (malheureusement courantes de l'avis même de certains soignants) me semble pertinent pour approfondir l'approchement des logiques de fonctionnement des agents de santé.

De la rationalisation des erreurs professionnelles... 

plupart des observations les ramènent dans un premier temps à leur inévitabilité en raison de la dimension humaine qui les caractérise : "l'erreur est humaine !" L'erreur médicale serait même, de l'avis de certains soignants, nécessaire pour enrichir l'expérience du métier.

Aussi, le souvenir des erreurs commises se cristallise sur les pratiques de débutants. À ce niveau, l'erreur professionnelle est définie par les soignants comme étant une inadéquation entre les connaissances théoriques acquises et la mise en pratique de celles-ci. L'erreur est alors associée à une maladresse de débutant.

'erreur est parfois associée à une inadéquation du diagnostic: que des symptômes décrits par un patient font prendre une décision de référence dans une autre structure de santé où l'on établit un diagnostic qui aurait pu être pris en charge dans la structure qui réfère, voilà qui est défini comme une erreur professionnelle. Dans cette situation, la reconnaissance de l'erreur chez le soignant s'accompagne d'une crainte de jugement d'incompétence par des pairs.

Dans la pratique, les erreurs sont souvent liées à une accumulation de fonctions : un infirmier prescrit souvent des soins que seul un médecin devrait poser selon les recommandations professionnelles. De telles pratiques sont "ordinaires" dans un pays où plus de $70 \%$ des médecins sont concentrés en milieu urbain. En milieu semi-urbain, le seul médecin est souvent débordé par des tâches administratives et arrive rarement à allier des sorties de supervisions à sa pratique médicale.

Le champ des erreurs est donc vaste et récurrent dans l'univers soignant. Comme pour atténuer l'impact de l'erreur, les soignants ont recours à la croyance et à la pratique religieuse. On explique alors l'erreur par le destin.

... à l'économie des sanctions

61 Face à l'ordinaire des fautes professionnelles, on est frappé par la quasi absence de la mise en application des sanctions édictées dans les lois. On pourrait, à bien des égards, parler d'économie de la sanction professionnelle dans le domaine de la santé. Du côté des usagers, les plaintes sont rares. L'observation des modalités d'organisation du fonctionnement des services laisse percevoir une superposition d'organisation associée à la succession et/ou à la mobilité des responsables des services de santé.

62 À l'échelle étatique, une diversité de structures est mise en place pour dit-on "lutter contre la corruption" : Commission nationale de lutte contre la fraude, Comité national d'éthique, Haute autorité de coordination de la lutte contre la corruption, Inspection général d'Etat, enquêtes parlementaires de l'Assemblée des Députés du Peuple ${ }^{17}$, etc. Mais en réalité, elles sont loin de fonctionner, en dépit des lois pléthoriques. Un intérêt socio-anthropologique pour la gestion des biens publics devrait aussi s'intéresser aux modes de fonctionnement de ces structures dont la lutte contre la corruption est affichée comme objectif principal.

La notion de faute professionnelle laisse entrevoir le hiatus entre l'éthique des professionnels de santé (apprise dans les écoles de formation) et les diverses dispositions (sociale, économique, religieuse) chez les agents de santé. Les conduites des agents de santé au cœur de l'espace public de la santé sont "gouvernées" par des dispositions qui débordent largement d'une éthique professionnelle. La dissociation entre l'univers social et l'univers professionnel est très mince. Le pouvoir des soignants s'exerce de facto par la reconnaissance d'une technique : l'art de soigner. Mais outre cet art technique, le pouvoir des professionnels de santé trouve sa légitimité dans la

Bulletin de l'APAD, 23-24 | 2002 
manipulation des codes sociaux (le don, la redistribution des biens, la notion d'aide). Si l'éthique professionnelle est déterminante pour un service public équitablement assuré, la restitution des résultats des études auprès des acteurs de la santé apparaît comme une passerelle indispensable pour engager une implication et une réflexion des soignants dans le cadre d'une amélioration de leurs services. La "bonne gouvernance" passe nécessairement par une analyse critique de la "gouvernance" en œuvre chez les professionnels de santé.

\section{BIBLIOGRAPHIE}

Aïch P., Fassin D., 1994, Les métiers de la santé. Enjeux de pouvoir et quête de légitimité, Paris, Editions Anthropos.

Becker H., 1962, "The nature of a profession", National Society for The Study of Education, Chicago : 27-46.

Blundo G. et Olivier de Sardan J.-P., 2001a, La corruption au quotidien en Afrique de l'Ouest : 94 $-167$.

Blundo G. et Olivier de Sardan J.-P., 2001b, "La corruption quotidienne en Afrique de l'Ouest", Politique Africaine, $\mathrm{n}^{\circ} 83:$ 8-37.

Bouju J., 2000, "Clientélisme, corruption et gouvernance locale à Mopti (Mali) ", Autrepart, n¹4 : 143-163.

Chauveau J.-P., Le Pape M., Olivier de Sardan J.-P., 2001, "La pluralité des normes et leurs dynamiques en Afrique" in Winter G. (ed.), Inégalités et politiques publiques en Afrique. Pluralité de normes et jeux d'acteurs, Paris, Karthala : 145-162.

Chevandier C., 1997, Les métiers de l'hôpital, Paris, ed. la Découverte, collection Repères.

Déchamp-Le Roux C., 1994, "Vocation médicale et choix professionnel", in Pierre Aïach et Didier Fassin (eds.), Les métiers de la santé. Enjeux de pouvoirs et quête de légitimité, Paris, Anthropos : 87-119.

Gobatto I., 1999, Etre médecin au Burkina Faso. Dissection sociologique d'une transplantation professionnelle, Paris, L'Harmattan.

Hahounou E.K., 2000, Etude socio-anthropologique des interactions entre usagers et agents de la santé. Le cas du service des urgences de l'Hôpital National de Niamey, DEA de Sciences Sociales, Marseille, EHESS.

Ki Z., 2002, Lois contre corruption. Inventaire des dispositions réglementaires et juridiques relatives à la lutte contre la corruption au Burkina Faso, Ouagadougou, REN-LAC.

L'Evénement, mars 2002, "Autopsie d'une gestion mafieuse. Surendettement crapuleux, laxisme d'Etat", (mensuel burkinabè) $n^{\circ} 10: 8-11$.

Médard J.-F., 1995, "La corruption politique et administrative et les différenciations du public et du privé : une perspective comparative" in Borghi M. et Meyer-Bisch P. (eds.), La corruption. L'envers des droits de l'homme, Fribourg, Les éditions universitaires : 37-46. 
Médard J.-F., 1998, "La crise de l'Etat néo-patrimonial et l'évolution de la corruption en Afrique sub-saharienne", Mondes en Développement, tome 26, $\mathrm{n}^{\circ} 102$ : 55-67.

Médard J.-F., 2000, "Clientélisme politique et corruption", Revue Tiers Monde, tome XLI, n 161 : 75-87.

Memmi A., 1979, La dépendance, Paris, Gallimard.

Memmi A., octobre 1980, "De la dépendance à la pourvoyance", Panorama du médecin, 1065.

Ministère de la santé, 1996, Statistiques sanitaires, Burkina Faso, Direction des études et de la planification.

Ministère de la santé, 1997, Carte sanitaire du Burkina Faso, Burkina Faso, Direction des études et de la planification.

Olivier de Sardan J.-P., 2001, "La sage-femme et le douanier, cultures professionnelles locales et culture bureaucratique privatisée", Autrepart (à paraître).

REN-LAC, 2001, Le Burkina à l'épreuve de la corruption, Ouagadougou, REN-LAC.

REN-LAC, 2002, Etat de la corruption au Burkina Faso, rapport 200 l, Ouagadougou, REN-LAC.

Rogoff N., 1957, "The decision to study medicine" in R. Merton, H. Reader and P. Kendall (ed.), The student Physician, Cambridge, Harvard Univ. Press : 109-129.

Sanou A., Bouda P., Ki-Zerbo L., Jacob J.-P., 2001, Morale et corruption dans les sociétés anciennes du Burkina (Bobo, Moaga, San et Winyé), Ouagadougou, REN-LAC.

\section{NOTES}

1.Dans un pays comme le Burkina Faso, on note une "banalisation" des pratiques de corruption par rapport aux années de la Révolution (1982-1987). D'un point de vue moral, on entend souvent dire que la corruption a assombri l'image traditionnelle du Burkina comme terre d'intégrité. C'est dans le même ordre d'idée qu'un ancien président de l'Assemblée des Députés du Peuple a pu déclarer que "la morale agonise au pays des hommes intègres". Selon le rapport 2001 du REN-LAC (Réseau National de Lutte Anti-Corruption) sur l'état de la corruption au Burkina. les structures publiques de santé sont des sphères où les pratiques de corruption sont "routinières" et "visibles". Les résultats de la même enquête (par sondage) classent le secteur de la santé selon l'importance du phénomène à la $7^{\mathrm{e}}$ place : marchés publics, police \& gendarmerie, administration publique, justice, douane, mairie, santé, impôts, média, enseignement.

2.La grande partie des matériaux de terrain de cet article provient d'une enquête réalisée de janvier à février 2001 au centre médical d'Orodara (Burkina Faso) dans le cadre d'un programme de recherche IRD-Union Européenne "Interactions entre les systèmes de Santé publique...". D'autres données ont été recueillies dans le cadre d'une étude en cours (2001-2003) soutenue par l'ANRS (Agence Nationale de Recherche sur le Sida).

3.Acronymes et abréviations utilisés dans le texte : CMA : Centre Médical avec Antenne chirurgicale ; Coges : Comité de gestion ; CSPS : Centre de Santé et de Promotion Sociale ; ECD : Equipe Cadre du District ; IDE : Infirmier Diplômé d'Etat ; IB : Infirmier 
Breveté ; MCD : Médecin Chef de District ; CHN ; Centre Hospitalier National ; CHR : Centre Hospitalier Régional.

4.De manière générale, les options universitaires choisies pendant l'année de préparation du baccalauréat sont rarement prises en compte. Par conséquent. seules la chance et les relations sociales décident souvent de l'orientation vers les sections choisies.

5.Terminologie pour désigner un homme qui exerce la profession de sage-femme. Ce néologisme utilisé à partir des années soixante-dix a été formé à partir du mot grec maïeutikê qui signifie "l'art de faire accoucher" (Chevandier 1997 : 94).

6.La pourvoyance est définie comme le désir de satisfaction consécutif aux soins médicaux procurés à autrui (Memmi 1979).

7. À Orodara, les frais d'hospitalisation sont de $200 \mathrm{~F}$ CFA (1 FF = $100 \mathrm{~F} \mathrm{CFA})$ et de $100 \mathrm{~F}$

CFA à partir du $15^{\mathrm{e}}$ jour d'hospitalisation. En revanche à Bobo-Dioulasso, les chambres d'hospitalisation sont classées par catégorie et le coût d'une journée d'hospitalisation peut atteindre $7000 \mathrm{~F}$ CFA. Les parents proches des soignants sont immédiatement classés dans la catégorie supérieure.

8.Le don, même dans les sociétés pré-coloniales, était rarement désintéressé.

9.L'enchâssement des pratiques de corruption dans les codes de sociabilité locaux dans des interactions entre professionnels et usagers des services de santé au Bénin et au Niger a été souligné dans le rapport sur La corruption au quotidien en Afrique de l'Ouest (Blundo et Olivier de Sardan 2001 : 94-167). Voir également Sanou \& al. 2001.

10.Voir également Blundo et Olivier de Sardan $2001: 30-31$.

11.Personnes vivant avec le VIH.

12.Le Centre Hospitalier National ( $\mathrm{CHN}$ ) est le niveau de soins le plus élevé. Sa vocation est de prendre en charge les patients dont le problème de santé n'a pu être résolu au niveau des échelons de soins précédents. Il est également un lieu de formation des différentes catégories de personnels et de recherche. Les CHN sont au nombre de deux : Ouagadougou et Bobo-Dioulasso (Ministère de la Santé. Carte Sanitaire du Burkina Faso. Direction des études et de la planification. 1997, page Il).

13.L'ECD (équipe cadre de district) est l'organe dirigeant du district. C'est l'ECD qui s'occupe de la planification, du suivi de l'évaluation des activités au niveau du district. L'ECD vient en appui des formations sanitaires. La planification, le suivi des vaccinations, c'est le rôle de l'ECD. Donc à partir des micros plans des formations sanitaires, l'ECD élabore son plan qui a une vision plus large. On peut faire partie de l'ECD pendant le service d'un MCD et se voir éjecter de "équipe avec l'arrivée d'un autre. Chaque MCD y apporte sa touche. Il n'y a pas de critère d'élection clairement établi pour faire partie de l'ECD ; le critère implicite de l'ancienneté est surtout doublé par celui de l'affinité avec le MCD.

14.Il est apparu aussi que le fait d'être un membre actif du parti politique. en l'occurrence le parti au pouvoir, constitue une source de revenu supplémentaire pour un soignant. C'est ainsi qu'on explique l'acquisition d'un bien de luxe. telle qu'une voiture, par un IDE. L'appartenance politique peut même conférer du pouvoir au sein de la structure de santé d'autant plus que les absences régulières d'un responsable politique seront moins perçues comme un manquement au devoir professionnelle qu'un attribut du pouvoir qu'il représente hors de l'hôpital.

15.Ce type de situation a également été remarqué par Isabelle Gobatto, op. cit. : 71. 16.Eric Hahonou (2000) a décrit d'une manière fine comment les interactions entre agents de santé et usagers au sein des urgences de l'Hôpital National de Niamey au 
Niger sont caractérisées par la violence exercée sur les usagers dans les relations qui ne sont définies ni par un réseau de connaissance ni par une transaction monétaire. Le paradoxe observable dans ce type de situations est celui d'un univers tantôt caractérisé par la "sur personnalisation" à l'égard d'usagers privilégiés (privilège d'appartenir à l'entregent ou d'avoir la capacité de corrompre). tantôt par la "déshumanisation" vis-à-vis des anonymes (Blundo \& Olivier de Sardan 2001a : 166).

17.Cf. Ki 2002.

\section{AUTEUR}

\section{FATOUMATA OUATTARA}

Allocataire de recherche à l'ANRS, chercheur associé à l'UR 002 "socio-anthropologie de la santé", IRD - SHADYC (Marseille). 